\title{
Leri-Weill Dyschondrosteosis
}

National Cancer Institute

\section{Source}

National Cancer Institute. Leri-Weill Dyschondrosteosis. NCI Thesaurus. Code C126560.

A bone growth disorder inherited in a pseudoautosomal dominant pattern caused by mutations in the SHOX gene. It is characterized by short long bones in the arms and legs, short stature, and abnormalities of the wrist and forearm bones which may cause pain and limit wrist movement. 